\title{
Synthesis and structure elucidation of hydrazones derived from $N$-(2,4-dimethylphenyl)-3-oxobutanamide
}

\section{Murat Çağlar Hamzaçebi, Sevim Rollas,* Ş. Güniz Küçükgüzel, and Bedia Koçyiğit Kaymakçığlu}

Marmara University, Faculty of Pharmacy, Department of Pharmaceutical Chemistry, Tibbiye cad. No: 49, 34668 Haydarpasa, Istanbul, Turkey.

E-mail:sevim@sevimrollas.com

\begin{abstract}
Diazonium salts derived from amines 1 (sulfanilic acid, 4-nitroaniline, 4-aminosalicylic acid, sulfanilamide, sulfamethoxazole, sulfathiazole, sulfapyridine, sulfamerazine) were coupled with $N$-(2,4-dimethylphenyl)-3-oxobutanamide (2) resulting in the formation of hydrazones $\mathbf{3 a}-\mathbf{h}$.
\end{abstract}

Keywords: Azo coupling, hydrazones

\section{Introduction}

Coupling products of diazonium salts with aliphatic active hydrogen compounds are widely used as intermediates for the synthesis of a large number of heterocyclic compounds. Pyrazoles, ${ }^{1,2}$ isoxazolone, ${ }^{3}$ 2-pyrazoline-5-one ${ }^{4,5}$ can be obtained by cyclization of coupling products with substituted hydrazine or hydroxylamine, respectively (Scheme 1). Both hydrazones ${ }^{5,6-9}$ and their cyclization compounds $^{2-4}$ possess important biological and pharmacological properties.

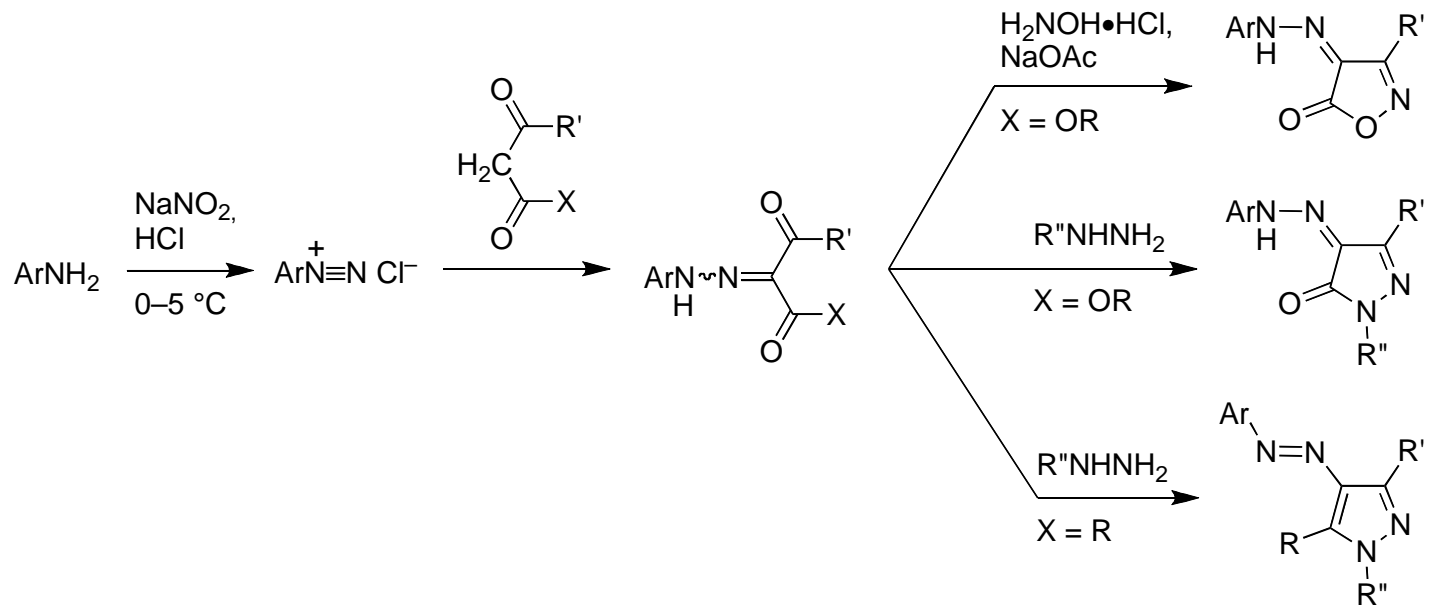

Scheme 1. General synthetic routes for the formation of azo compounds and hydrazones. 
Azo dyes are among the most important synthetic coloring agents but are regarded as potential carcinogens ${ }^{10}$ due to their metabolism; the reduction of the azo group affects their toxicity, mutagenicity, and carcinogenicity. ${ }^{10-13}$ Hydrazone dyes are considered non-genotoxic and non-mutagenic; e.g., 2-[(2-methoxy-5-nitrophenyl)hydrazono]- $N$-(2-methoxyphenyl)-3-oxobutanamide (PY74) is a hydrazone pigment used in yellow tattoo inks. The metabolism of PY $74^{14}$ and compounds containing azo group ${ }^{15,16}$ has been investigated using rat liver and human liver microsomes.

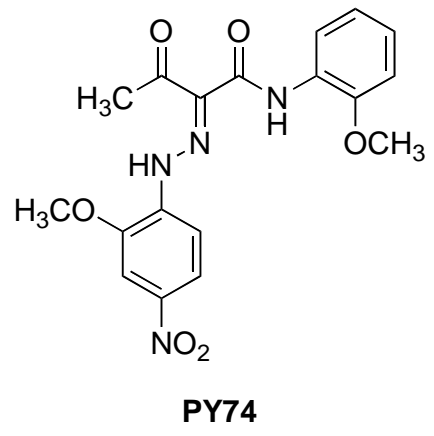

The hydrazone product obtained by azo coupling of the diazonium salt of sulfapyridine with $N$-(2-methylphenyl)-3-oxobutanamide has been found to be an HIV integrase inhibitor. ${ }^{17}$

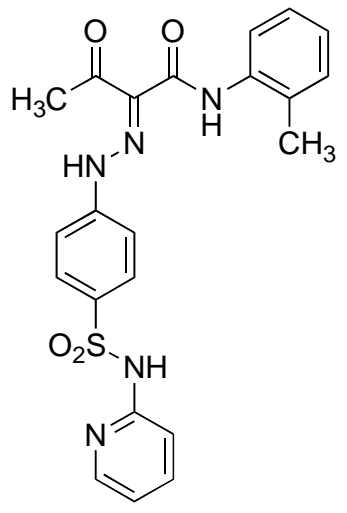

Earlier reports indicate that diazonium salts of certain aromatic amines such as sulfaguanidine ${ }^{18}$ and sulfanilamide ${ }^{19}$ have been coupled with compounds possessing active hydrogen. Furthermore, sulfanilamide derivatives have been reported to possess antibacterial activity. ${ }^{20}$ The present study reports on the synthesis of new coupling products $\mathbf{3 a}-\mathbf{h}$, which were obtained from diazonium salts derived from amines 1 (sulfanilic acid, 4-nitroaniline, 4-aminosalicylic acid, sulfanilamide, sulfamethoxazole, sulfathiazole, sulfapyridine, sulfamerazine) with $N$-(2,4-dimethylphenyl)-3-oxobutanamide (2). 


\section{Results and Discussion}

The diazonium salts derived from anilines 1 (sulfanilic acid, 4-nitroaniline, 4-aminosalicylic acid, sulfanilamide, sulfamethoxazole, sulfathiazole, sulfapyridine, sulfamerazine) were coupled with $\mathrm{N}$-(2,4-dimethylphenyl)-3-oxobutanamide (2) in aqueous ethanol containing sodium acetate resulting in the formation of hydrazones $\mathbf{3 a}-\mathbf{h}$ (Scheme 2).

The UV spectra of products 3 show three ranges of absorption maxima at 203-207, 235-271, and 376-393 nm, except compounds $\mathbf{3 e}$ and $\mathbf{3 f}$ which had four absorption maxima. Absorption bands attributed to an azo function between $332-360 \mathrm{~nm}^{21-22}$ and above $400 \mathrm{~nm}^{23}$ are missing. Thus, the observation of bands at $376-393 \mathrm{~nm}$ is indicative of the hydrazone form of compounds 3a-h. ${ }^{6,9,24-26}$

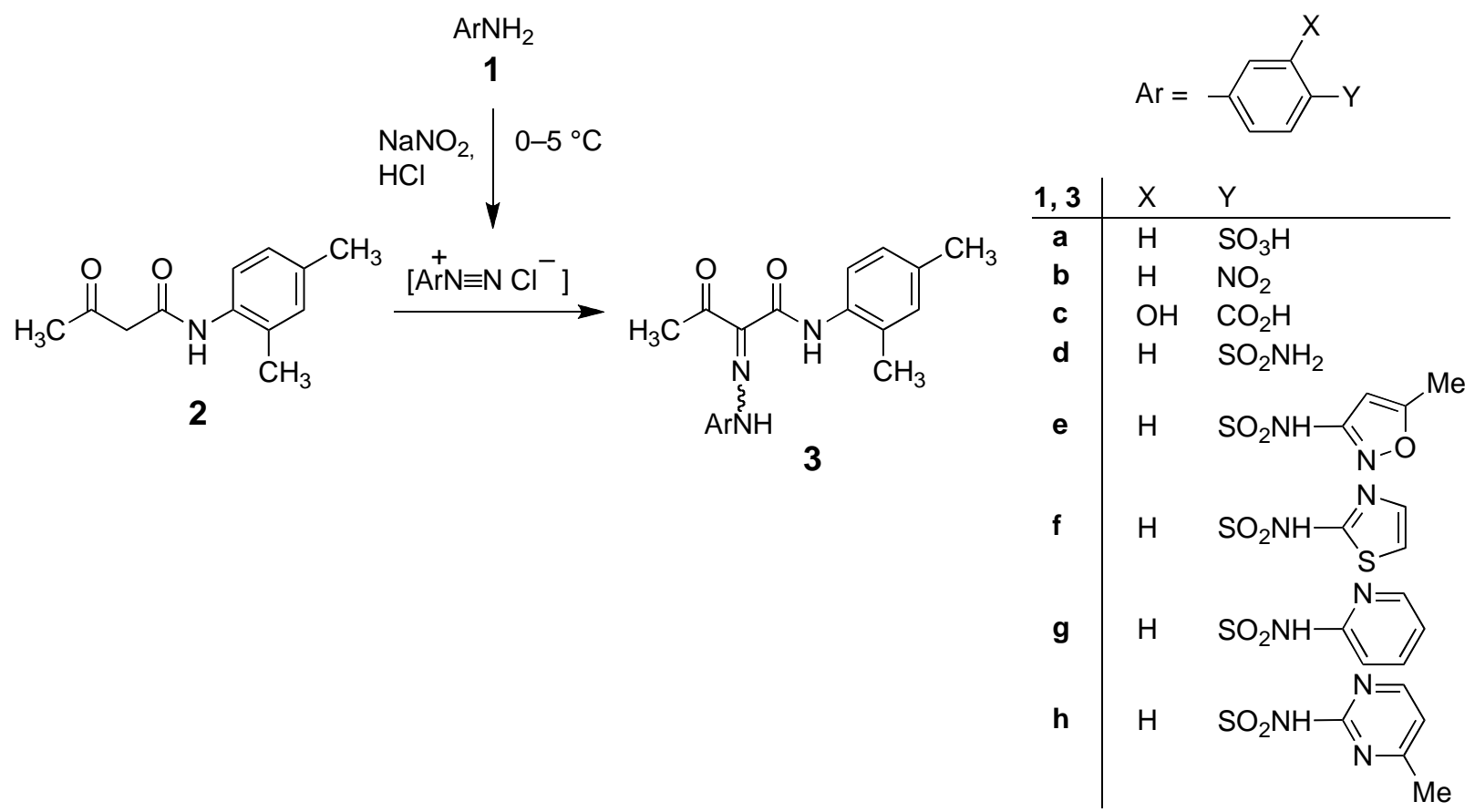

Scheme 2. Preparation of [2-[1-(2,4-dimethylphenylamino)-1,3-dioxobutan-2-ylidene]hydrazinyl]benzenes (3a-h).

The amide proton $(-\mathrm{NH}-\mathrm{C}=\mathrm{O})$ exhibits a singlet at $\delta 10.98-11.16$, the hydrazone proton $(-\mathrm{CH}=\mathrm{N}-\mathrm{NH}-)$ shows a singlet at $\delta 13.90-14.32$; both signal ranges are in agreement with the literature. ${ }^{18,27-29}$ Furthermore, in the ${ }^{1} \mathrm{H}-\mathrm{NMR}$ spectra of compounds $\mathbf{3 a - h}$, signals arising from a $>\mathrm{CH}-\mathrm{N}=\mathrm{N}$ - moiety are expected at $3.00-4.00 \mathrm{ppm},{ }^{23,25}$ but were not observed. These findings support the hydrazone structure of the products.

The APCI-MS spectra of $\mathbf{3 a}-\mathbf{h}$ show molecular ion peaks $\left(\mathrm{M}^{+}\right)$confirming their molecular weight; common characteristic fragment ions result from cleavage of the amide bond resulting in 
2,4-dimethylanilinium ion $(m / z \quad 122)$ and the complementing 2-[(2-arylhydrazono)-3-oxobutylidyne]oxonium ion $(\mathrm{m} / \mathrm{z} \mathrm{M}+1-122)$.

\section{Experimental Section}

General Procedures. All chemicals and solvents were commercially acquired. Melting points were determined with a Schmelzpunktbestimmer SMP II. The UV spectra were measured with a Shimadzu UV-2100 S. The IR spectra were obtained with a Shimadzu FTIR-8400. ${ }^{1} \mathrm{H}-\mathrm{NMR}$ spectra in DMSO- $d_{6}$ were recorded on a Bruker Avance-DPX-400 spectrometer $(400 \mathrm{MHz})$. APCI-MS was performed using an Agilent 1100 MSD spectrometer at $100 \mathrm{eV}$ (positive polarity). All new compounds were analyzed for C, H, N (Leco CHNS 932).

${ }^{1} \mathrm{H}$ NMR, APCI-MS, and elemental analyses were provided by the Scientific and Technical Research Council of Turkey, (TÜBITAK).

\section{[2-[1-(2,4-Dimethylphenylamino)-1,3-dioxobutan-2-ylidene]hydrazinyl]benzenes}

$(3 \mathbf{a}-\mathbf{h})$.

\section{General procedure}

To the cooled $\left(0-5{ }^{\circ} \mathrm{C}\right)$ solution of amine $1(0.01 \mathrm{~mol})$ in ethanol $(50 \mathrm{~mL})$ and hydrochloric acid $(4 \% ; 40 \mathrm{~mL})$ was added an ice-cold solution of sodium nitrite $(7 \% ; 10 \mathrm{~mL})$. The reaction mixture was then poured into a solution of $N$-(2,4-dimethylphenyl)-3-oxo-butanamide $(2 ; 2.05 \mathrm{~g}$, $0.01 \mathrm{~mol})$ and sodium acetate $(60 \mathrm{~g}, 0.73 \mathrm{~mol})$ in ethanol $(50 \% ; 50 \mathrm{~mL})$ under vigorous stirring. The precipitated solid was collected, washed with water, air-dried at room temperature, and washed with ethanol to give 3 .

${ }^{1} \mathrm{H}$ NMR spectra: H' refers to the X,Y-substituted benzene ring Ar (cf. Scheme 2).

\section{4-[2-[1-(2,4-Dimethylphenylamino)-1,3-dioxobutan-2-ylidene]hydrazinyl]benzenesulfonic}

acid (3a). Yellow needles (3.46 g, 89\%); mp $357^{\circ} \mathrm{C}$ (EtOH; decomp.). UV $\lambda_{\max }$ (EtOH): $\mathrm{nm}$ $(\log \varepsilon) 376$ (4.53), 250 (4.26), 204 (4.45). IR (KBr): $v_{\text {max. }}\left(\mathrm{cm}^{-1}\right)$ 3592, 3511 (OH), 3170-3130 $(\mathrm{NH}), 1680$ (ketone $\mathrm{C}=\mathrm{O}), 1600$ (amide $\mathrm{C}=\mathrm{O}), 1315-1135(\mathrm{~S}=\mathrm{O}) .{ }^{1} \mathrm{H}$ NMR (DMSO- $\left.d_{6}\right): \delta 2.26$ $\left(3 \mathrm{H}, \mathrm{s}, \mathrm{CH}_{3}\right), 2.29\left(3 \mathrm{H}, \mathrm{s}, \mathrm{CH}_{3}\right), 2.55\left(3 \mathrm{H}, \mathrm{s}, \mathrm{COCH}_{3}\right), 7.04\left(1 \mathrm{H}, \mathrm{d},{ }^{3} J_{6,5}=8.2 \mathrm{~Hz}, \mathrm{H} 6\right), 7.11(1 \mathrm{H}$, s, H3), 7.45-7.70 (4H, m, H2', H3', H5', H6'), $7.96\left(1 \mathrm{H}, \mathrm{d},{ }^{3} J_{5,6}=8.2 \mathrm{~Hz}, \mathrm{H} 5\right), 11.16(1 \mathrm{H}, \mathrm{s}$, CONH), 14.32 (1H, s, NNH). APCI-MS: $m / z$ (\%) 390 (100) [M+1] $]^{+}, 371$ (3.7), 269 (24), 152 (4.4), 122 (37). Anal. calcd. for $\mathrm{C}_{18} \mathrm{H}_{19} \mathrm{~N}_{3} \mathrm{O}_{5} \mathrm{~S} \cdot 2 \mathrm{H}_{2} \mathrm{O}$ : C, 50.81; H, 5.45; N, 9.88; S, 7.54. Found: C, 50.25; H, 5.24; N, 10.03; S, 7.23.

$\mathrm{N}$-(2,4-Dimethylphenyl)-2-[2-(4-nitrophenyl)hydrazono]-3-oxobutanamide (3b). Yellow needles (2.76 g, 78\%); mp $222{ }^{\circ} \mathrm{C}(\mathrm{EtOH}) . \mathrm{UV} \lambda_{\max }(\mathrm{EtOH}): \mathrm{nm}(\log \varepsilon) 392$ (4.40), 235 (4.06), 203 (4.34). IR (KBr): $v_{\max }\left(\mathrm{cm}^{-1}\right): 3220,3130(\mathrm{NH}), 1663$ (ketone $\left.\mathrm{C}=\mathrm{O}\right), 1595$ (amide $\left.\mathrm{C}=\mathrm{O}\right) .{ }^{1} \mathrm{H}$ NMR (DMSO-d $\left.)_{6}\right): \delta 27\left(3 \mathrm{H}, \mathrm{s}, \mathrm{CH}_{3}\right), 2.29\left(3 \mathrm{H}, \mathrm{s}, \mathrm{CH}_{3}\right), 2.57\left(3 \mathrm{H}, \mathrm{s}, \mathrm{COCH}_{3}\right), 7.05(1 \mathrm{H}, \mathrm{d}$, $\left.{ }^{3} J_{6,5}=8.8 \mathrm{~Hz}, \mathrm{H} 6\right), 7.11(1 \mathrm{H}, \mathrm{s}, \mathrm{H} 3), 7.71-8.30(5 \mathrm{H}, \mathrm{m}, \mathrm{H} 5, \mathrm{H} 2$ ', H3', H5', H6'), 11.00 (1H, s, CONH), 13.90 (1H, s, NNH). APCI-MS: m/z (\%) 355 (100) [M+1] 234 (31.5), 139 (5.0), 122 
(39.1). Anal. calcd. for $\mathrm{C}_{18} \mathrm{H}_{18} \mathrm{~N}_{4} \mathrm{O}_{4}$ : C, 61.01; H, 5.12; N, 15.81. Found: C, 60.71; H, 4.98; N, 16.21 .

4-[2-[1-(2,4-Dimethylphenylamino)-1,3-dioxobutan-2-ylidene]hydrazinyl]-2-hydroxybenzoic acid (3c). Yellow needles (2.92 g, 79\%); mp $245^{\circ} \mathrm{C}(\mathrm{EtOH})$. UV $\lambda_{\max .}(\mathrm{EtOH}): \mathrm{nm}(\log \varepsilon) 386$ (4.55), 271 (4.24), 206 (4.60). IR (KBr): $v_{\max }\left(\mathrm{cm}^{-1}\right): 3220,3130(\mathrm{NH}), 1680$ (carboxylic acide $\mathrm{C}=\mathrm{O}), 1660$ (ketone $\mathrm{C}=\mathrm{O}), 1638$ (amide $\mathrm{C}=\mathrm{O}) .{ }^{1} \mathrm{H}$ NMR (DMSO- $\left.d_{6}\right): \delta 2.26\left(3 \mathrm{H}, \mathrm{s}, \mathrm{CH}_{3}\right), 2.28$ $\left(3 \mathrm{H}, \mathrm{s}, \mathrm{CH}_{3}\right), 2.54\left(3 \mathrm{H}, \mathrm{s}, \mathrm{COCH}_{3}\right), 7.00(1 \mathrm{H}, \mathrm{s}, \mathrm{H} 2), 7.01\left(1 \mathrm{H}, \mathrm{d},{ }^{3} J_{6,5}=8.0 \mathrm{~Hz}, \mathrm{H} 6\right), 7.05(1 \mathrm{H}$, $\left.\mathrm{d},{ }^{3} J_{6^{\prime}, 5^{\prime}}=10.0 \mathrm{~Hz}, \mathrm{H} 6^{\prime}\right), 7.09(1 \mathrm{H}, \mathrm{s}, \mathrm{H} 3), 7.79\left(1 \mathrm{H}, \mathrm{d},{ }^{3} J_{5^{\prime}, 6^{\prime}}=9.2 \mathrm{~Hz}, \mathrm{H} 5^{\prime}\right), 7.93\left(1 \mathrm{H}, \mathrm{d},{ }^{3} J_{5,6}=\right.$ $8.2 \mathrm{~Hz}, \mathrm{H} 5), 11.04(1 \mathrm{H}, \mathrm{s}, \mathrm{CONH}), 13,90(1 \mathrm{H}, \mathrm{s}, \mathrm{NNH})$. APCI-MS: $m / z(\%): 370(100)[\mathrm{M}+1]^{+}$, 355 (16.6), 249 (53.9), 122 (23.6). Anal. calcd. for $\mathrm{C}_{19} \mathrm{H}_{19} \mathrm{~N}_{3} \mathrm{O}_{5} . \mathrm{H} 2 \mathrm{O}: \mathrm{C}, 58.91 ; \mathrm{H}, 5.46 ; \mathrm{N}$, 10.85.Found: C, 58.73; H, 4.73; N, 11.12.

$\mathbf{N - ( 2 , 4 - D i m e t h y l p h e n y l ) - 3 - o x o - 2 - [ 2 - ( 4 - s u l f a m o y l p h e n y l ) h y d r a z o n o ] b u t a n a m i d e ~ ( 3 d ) . ~}$

Yellow needles $(3.06 \mathrm{~g}, 79 \%) ; \mathrm{mp} 260{ }^{\circ} \mathrm{C}(\mathrm{EtOH})$. UV $\lambda_{\max .}(\mathrm{EtOH}): \mathrm{nm}(\log \varepsilon) 376$ (4.17), 236 (3.92), 203 (4.33). IR (KBr): $v_{\max }\left(\mathrm{cm}^{-1}\right): 3317,3234,3170(\mathrm{NH}), 1663$ (ketone $\left.\mathrm{C}=\mathrm{O}\right), 1596$ (amide $\mathrm{C}=\mathrm{O}), 1151(\mathrm{~S}=\mathrm{O}) .{ }^{1} \mathrm{H}$ NMR (DMSO $\left.d_{6}\right): \delta 2.27\left(3 \mathrm{H}, \mathrm{s}, \mathrm{CH}_{3}\right), 2.29\left(3 \mathrm{H}, \mathrm{s}, \mathrm{CH}_{3}\right), 2.57$ $\left(3 \mathrm{H}, \mathrm{s}, \mathrm{COCH}_{3}\right), 7.05\left(1 \mathrm{H}, \mathrm{d},{ }^{3} J_{6,5}=8.2 \mathrm{~Hz}, \mathrm{H} 6\right), 7.10(1 \mathrm{H}, \mathrm{s}, \mathrm{H} 3), 7,34\left(1 \mathrm{H}, \mathrm{s}, \mathrm{SO}_{2} \mathrm{NH}\right), 7.66-$ 7.90 (4H, m, H2', H3', H5', H6'), 7.94 (1H, d, $\left.{ }^{3} J_{5,6}=8.2 \mathrm{~Hz}, \mathrm{H} 5\right), 11.05$ (1H, s, CONH), 14.10 (1H, s, NNH). APCI-MS: $m / z(\%): 389$ (28.2) [M+1] $]^{+}, 372$ (7.3), 283 (43.9), 268 (91.6), 122 (100). Anal. calcd. for $\mathrm{C}_{18} \mathrm{H}_{20} \mathrm{~N}_{4} \mathrm{O}_{4} \mathrm{~S}$ : C, 55.66; H, 5.19; N, 14.42; S, 8.25. Found: C, 55.83; H, 5.26; N, 14.19; S, 7.84.

$N$-(2,4-Dimethylphenyl)-2-[2-[4-[ $N$-(5-methylisoxazol-3-yl)sulfamoyl]phenyl]hydrazono]-3oxobutanamide (3e). Yellow needles $(4.03 \mathrm{~g}, 86 \%)$; mp $215{ }^{\circ} \mathrm{C}(\mathrm{EtOH})$. UV $\lambda_{\max }(\mathrm{EtOH}): \mathrm{nm}$ (log ع) 376 (4.44), 249 (4.15), 237 (4.16), 204 (8.98). IR (KBr): $v_{\max }\left(\mathrm{cm}^{-1}\right): 3220,3130(\mathrm{NH})$, 1667 (ketone $\mathrm{C}=\mathrm{O}), 1614$ (amide $\mathrm{C}=\mathrm{O}), 1137(\mathrm{~S}=\mathrm{O}) .{ }^{1} \mathrm{H}$ NMR $\left(\mathrm{DMSO}-d_{6}\right): \delta 2.26\left(3 \mathrm{H}, \mathrm{s}, \mathrm{CH}_{3}\right)$, $2.28\left(3 \mathrm{H}, \mathrm{s}, \mathrm{CH}_{3}\right), 2.30\left(3 \mathrm{H}, \mathrm{s}, \mathrm{CH}_{3}\right.$ at isoxazole $), 2.55\left(3 \mathrm{H}, \mathrm{s}, \mathrm{COCH}_{3}\right), 6.15(1 \mathrm{H}, \mathrm{s}, \mathrm{CH}$ isoxazole), $7.04\left(1 \mathrm{H}, \mathrm{d},{ }^{3} J_{6,5}=8.3 \mathrm{~Hz}, \mathrm{H} 6\right), 7.10(1 \mathrm{H}, \mathrm{s}, \mathrm{H} 3), 7.67-7.92(4 \mathrm{H}, \mathrm{m}, \mathrm{H} 2$ ', H3', H5', H6'), $7.92\left(1 \mathrm{H}, \mathrm{d},{ }^{3} J_{5,6}=8.2 \mathrm{~Hz}, \mathrm{H} 5\right), 10.98(1 \mathrm{H}, \mathrm{s}, \mathrm{CONH}), 11.40\left(1 \mathrm{H}, \mathrm{s}, \mathrm{SO}_{2} \mathrm{NH}\right), 14.02(1 \mathrm{H}, \mathrm{s}$, NNH). APCI-MS: $m / z(\%) 470$ (100) [M+1] $]^{+}, 372$ (19.6), 349 (48.7), 254 (26.5), 122 (22.0). Anal. calcd. for $\mathrm{C}_{22} \mathrm{H}_{23} \mathrm{~N}_{5} \mathrm{O}_{5} \mathrm{~S}: \mathrm{C}, 56.28 ; \mathrm{H}, 4.94 ; \mathrm{N}, 14.92 ; \mathrm{S}, 6.83$. Found: C, 56.19; H, 4.52; N, $14.98 ; \mathrm{S}, 6.63$.

$N$-(2,4-Dimethylphenyl)-3-oxo-2-[2-[4-( $N$-thiazol-2-ylsulfamoyl)phenyl]hydrazono]butanamide (3f). Yellow needles $(3.34 \mathrm{~g}, 71 \%) ; \mathrm{mp} 280^{\circ} \mathrm{C}(\mathrm{EtOH}) . \mathrm{UV} \lambda_{\max }(\mathrm{EtOH}): \mathrm{nm}(\log \varepsilon) 381$ (4.31), 271 (4.08), 251 (4.03), 202 (4.39). IR (KBr): $v_{\max .}\left(\mathrm{cm}^{-1}\right): 3220,3130(\mathrm{NH}), 1665$ (ketone $\mathrm{C}=\mathrm{O}), 1630$ (amide $\mathrm{C}=\mathrm{O}), 1149(\mathrm{~S}=\mathrm{O}) .{ }^{1} \mathrm{H}-\mathrm{NMR}\left(\mathrm{DMSO}-d_{6}\right): \delta 2.26\left(3 \mathrm{H}, \mathrm{s}, \mathrm{CH}_{3}\right), 2.28(3 \mathrm{H}, \mathrm{s}$, $\left.\mathrm{CH}_{3}\right), 2.55\left(3 \mathrm{H}, \mathrm{s}, \mathrm{COCH}_{3}\right), 6.84\left(1 \mathrm{H}, \mathrm{d},{ }^{3} J_{5,4}: 4.5 \mathrm{~Hz}, \mathrm{H} 5\right.$ thiazole $), 7.03\left(1 \mathrm{H}, \mathrm{d},{ }^{3} J_{6,5}=8.3 \mathrm{~Hz}\right.$, H6), $7.09(1 \mathrm{H}, \mathrm{s}, \mathrm{H} 3), 7.26\left(1 \mathrm{H}, \mathrm{d},{ }^{3} J_{4,5}=4.6 \mathrm{~Hz}, \mathrm{H} 4\right.$ thiazole), 7.62-7.88 (4H, m H2', H3', H5', H6'), $7.93\left(1 \mathrm{H}, \mathrm{d},{ }^{3} J_{5,6}=8.2 \mathrm{~Hz}, \mathrm{H} 5\right), 11.03(1 \mathrm{H}, \mathrm{s}, \mathrm{CONH}), 12.75\left(1 \mathrm{H}, \mathrm{s}, \mathrm{SO}_{2} \mathrm{NH}\right), 14,11(1 \mathrm{H}$, s, NNH). APCI-MS: $m / z(\%) 472(100)[\mathrm{M}+1]^{+}, 372$ (9.5), 351 (17.0), 256 (10.9), 122 (7.3). Anal. calcd. for $\mathrm{C}_{21} \mathrm{H}_{21} \mathrm{~N}_{5} \mathrm{O}_{4} \mathrm{~S}_{2} \cdot 1 / 2 \mathrm{H}_{2} \mathrm{O}$ : C, 52.50; H, 4.58; N, 14.58; S, 13.33. Found: C, 52.13; H, 5.14; N, 14.83; S, 13.38 . 
N-(2,4-Dimethylphenyl)-3-oxo-2-[2-[4-( $N$-pyridin-2-ylsulfamoyl)phenyl]hydrazono]butanamide (3g). Yellow needles $(3.12 \mathrm{~g}, 67 \%) ; \mathrm{mp} 240{ }^{\circ} \mathrm{C}(\mathrm{EtOH})$. UV $\lambda_{\max }(\mathrm{EtOH}): \mathrm{nm}(\log \varepsilon)$ : 375 (4.11), 247 (3.93), 203 (4.31). IR (KBr): $v_{\max .}\left(\mathrm{cm}^{-1}\right)$ : 3220, $3130(\mathrm{NH}), 1668$ (ketone $\left.\mathrm{C}=\mathrm{O}\right)$, 1633 (amide $\mathrm{C}=\mathrm{O}), 1139(\mathrm{~S}=\mathrm{O}) .{ }^{1} \mathrm{H}$ NMR (DMSO- $\left.d_{6}\right): \oint 2.26\left(3 \mathrm{H}, \mathrm{s}, \mathrm{CH}_{3}\right), 2.28\left(3 \mathrm{H}, \mathrm{s}, \mathrm{CH}_{3}\right)$, $2.54\left(3 \mathrm{H}, \mathrm{s}, \mathrm{COCH}_{3}\right), 6.88(1 \mathrm{H}, \mathrm{t}, \mathrm{H} 4$ pyridine $), 7.03\left(1 \mathrm{H}, \mathrm{d},{ }^{3} \mathrm{~J}_{6,5}=8.7 \mathrm{~Hz}, \mathrm{H} 6\right), 7.09(1 \mathrm{H}, \mathrm{s}, \mathrm{H} 3)$, $7.16\left(1 \mathrm{H}, \mathrm{d},{ }^{3} J_{5,6}=8.3 \mathrm{~Hz}, \mathrm{H} 5\right.$ pyridine $), 7.62-7.93$ (5H, m, H3 pyridine, H2', H3', H5', H6'), 7.97 $\left(1 \mathrm{H}, \mathrm{d},{ }^{3} J_{5,6}=8.3 \mathrm{~Hz}, \mathrm{H} 5\right), 8.01\left(1 \mathrm{H}, \mathrm{d},{ }^{3} J_{6,5}=4.4 \mathrm{~Hz}, \mathrm{H} 6\right.$ pyridine $), 11.02(1 \mathrm{H}, \mathrm{s}, \mathrm{CONH}), 11.90$ $\left(1 \mathrm{H}, \mathrm{s}, \mathrm{SO}_{2} \mathrm{NH}\right), 14.08$ (1H, s, NNH). APCI-MS: $m / z 466$ (100) [M+1] $]^{+}, 345$ (3.6), 318 (1.4), 268 (2.2), 250 (4.2), 122 (4.1). Anal. calcd. for $\mathrm{C}_{23} \mathrm{H}_{23} \mathrm{~N}_{5} \mathrm{O}_{4} \mathrm{~S} \cdot \mathrm{H}_{2} \mathrm{O}$ : C, 57.13; H, 5.21; N, 14.48; S, 6.63. Found: C, 57.95; H, 4.81; N, 14.81; S, 6.85.

$\boldsymbol{N}$-(2,4-Dimethylphenyl)-2-[2-[4-[ $N$-(4-methylpyrimidin-2-yl)sulfamoyl]phenyl]hydrazono]3-oxobutanamide (3h). Yellow needles $(3.02 \mathrm{~g}, 63 \%)$; mp $288{ }^{\circ} \mathrm{C}$. UV $\lambda_{\max .}(\mathrm{EtOH}): \mathrm{nm}(\log \varepsilon)$ 376 (4.49), 253 (4.28), 203 (4.53). IR (KBr): $v_{\max }\left(\mathrm{cm}^{-1}\right)$ : 3250, $3120(\mathrm{NH}), 1668$ (ketone $\left.\mathrm{C}=\mathrm{O}\right)$, 1615 (amide $\mathrm{C}=\mathrm{O}), 1343,1175(\mathrm{~S}=\mathrm{O}) .{ }^{1} \mathrm{H}$ NMR (DMSO-d $)$ : $\delta .2 .26\left(3 \mathrm{H}, \mathrm{s}, \mathrm{CH}_{3}\right), 2.28(3 \mathrm{H}, \mathrm{s}$, $\left.\mathrm{CH}_{3}\right), 2.33\left(3 \mathrm{H}, \mathrm{s}, \mathrm{CH}_{3}\right.$ at pyrimidine), $2.55\left(3 \mathrm{H}, \mathrm{s}, \mathrm{COCH}_{3}\right), 6.90\left(1 \mathrm{H}, \mathrm{d},{ }^{3} J_{6,5}=5.0 \mathrm{~Hz}, \mathrm{H} 6\right.$ pyrimidine), $7.00\left(1 \mathrm{H}, \mathrm{d},{ }^{3} J_{6,5}=8.2 \mathrm{~Hz}, \mathrm{H} 6\right), 7.09$ (1H, s, H3), 7.65-8.06 (5H, m H5, H2', H3', H5', H6'), $8.32\left(1 \mathrm{H}, \mathrm{d},{ }^{3} J_{5,6}=5.0 \mathrm{~Hz}, \mathrm{H} 5\right.$ pyrimidine), $11.01(1 \mathrm{H}, \mathrm{s}, \mathrm{CONH}), 11.67(1 \mathrm{H}, \mathrm{s}$, $\left.\mathrm{SO}_{2} \mathrm{NH}\right), 14.07$ (1H, s, NNH). APCI-MS: $m / z$ (\%) 481 (100) [M+1] $]^{+}, 467$ (4.0), 332 (6.3), 265 (4.1). Anal. calcd. for $\mathrm{C}_{23} \mathrm{H}_{24} \mathrm{~N}_{6} \mathrm{O}_{4} \mathrm{~S}$ : C, 57.49; H, 5.03; N, 17.49; S, 6.67. Found: C, 57.36; H, 5.73; N, 17.67; S, 6.59.

\section{Acknowledgements}

This study was supported by Scientific Research Project Commission of Marmara University (Project number: SAĞ-YLS-290506-0086).

\section{References}

1. Ergenç, N.; Rollas, S. J. Fac. Pharm. Istanbul 1975, 11, 138; Chem. Abstr. 1977, 87, 68222 .

2. Oruç, E. E.; Koçyiğit-Kaymakçıŏ̆lu, B.; Oral, B.; Altunbaş-Toklu, H. Z.; Kabasakal, L.; Rollas, S. Arch. Pharm. Chem. Life Sci. 2006, 339, 267.

3. Kömürcü, Ş. G.; Rollas, S.; Yılmaz, N.; Çevikbaş, A. Drug Metab. Drug Interact. 1995, 12, 161. Chem. Abstr. 1996, 124, 117186.

4. Küçükgüzel, Ş. G.; Rollas, S.; Erdeniz, H.; Kiraz, M.; Ekinci, A. C.; Vidin, A. Eur. J. Med. Chem. 2000, 35, 761.

5. Küçükgüzel, Ş. G.; Rollas, S. Farmaco 2002, 57, 583.

6. Küçükgüzel, Ş. G.; Rollas, S.; Erdeniz, H.; Kiraz M. Eur. J. Med. Chem. 1999, 34, 153. 
7. Küçükgüzel, Ş. G.; Rollas, S.; Küçükgüzel, İ.; Kiraz, M. Eur. J. Med. Chem. 1999, 34, 1093.

8. Kaymakçıŏlu, K. B.; Rollas, S. Farmaco 2002, 57,595.

9. Tsai, W. J., Shiao, Y. J., Lin, S. J., Chiou, W. F., Lin, L. C., Yang, T. H., Teng, C. M., Wu, T. S., Yang, L. M. Bioorg. Med. Chem. Lett. 2006, 16, 4440.

10. Golka, K.; Kopps, S.; Myslak, Z. W. Tox. Lett. 2004, 151, 203.

11. Moller, P.; Wallin, H. Mutat. Res. 2000, 462, 13.

12. Mansour, H. B.; Corroler, D.; Barillier, D.; Ghedira, K.; Chekir, L.; Mosrati, R. Food Chem.. Toxicol. 2007, 45, 1670.

13. Levine, W. G. Drug Metab. Rev. 1991, 23, 253.

14. Cui, Y.; Churchwell, M. I.; Couch, L. H.; Doerge, D. R.; Howard, P. C. Drug Metab. Dispos. 2005, 33, 1459.

15. Bekçe, B.; Şener, G.; Oktav, M.; Ülgen, M.; Rollas, S. Eur. J. Drug Metabol. Pharmacokinet. 2005, 30, 91.

16. Koçyiğit-Kaymakçıŏ̆lu, B.; Oruç, E. E.; Ünsalan, S.; Kabasakal, L.; Rollas, S. J. Chromatogr. B 2006, 831, 184.

17. Scozzafava, A.; Owa, T.; Mastrolorenzo, A.; Supuran, C. T. Curr. Med. Chem. 2003, 10, 925.

18. Ergenç, N.; Rollas, S. J. Fac. Pharm. Ístanbul 1974, 10, 77; Chem. Abstr. 1975, 82, 140004.

19. Ergenç, N.; Salman, A.; Gürsoy, A.; Bankaoğlu, G. Pharmazie 1990, 45, 346.

20. Ravichandran, V.; Mohan, S.; Kumar, K. S. Arkivoc 2007, xiv, 51.

21. Malik, W. U.; Garg, H. G.; Arora, V. J. Pharm. Sci. 1971, 60, 1738.

22. Garg, H. G.; Arora, V. J. Pharm. Sci. 1972, 61, 130.

23. Elguero, J.; Jacquier, R.; Tarrago, G. Bull. Soc. Chim. France 1966, 9, 2981.

24. Ergenç, N.; Rollas, S.; Demir, S.; Özdemir, F. J. Fac. Pharm. Istanbul 1975, 11, 183 ; Chem. Abstr. 1977, 87, 68224.

25. Ergenç, N.; Rollas, S. J. Fac. Pharm. İstanbul 1975, 11, 8; Chem. Abstr. 1975, 83, 192708.

26. Pabuççuoğlu, V.; Rollas, S. J. Fac. Pharm. Mar. 1991, 7, 39; Chem. Abstr. 1992, 117, 90214.

27. Ergenç, N.; Demir, S.; Rollas, S.; Özdemir, F.; Özçekiç, H. J. Fac. Pharm. Istanbul 1976, 12, 132; Chem. Abstr. 1977, 87, 134291.

28. Nakpathom, M.; Hinks, D.; Freeman, H. S. Dyes and Pigments 2001, 48, 93.

29. Garnovskii, A. D.; Ureav, A. I.; Minkin, V. I. Arkivoc 2004, iii, 29. 